\title{
EXPRESIÓN SEXUAL DE LAS PERSONAS CON TEA: PERCEPCIÓN DE LOS PROFESIONALES DE LA EDUCACIÓN
}

\section{Sexual expression of people with ASD: perception of education professionals}

\author{
Vanesa Lancha Villamayo \\ Universidad del País Vasco. \\ Departamento de Métodos de Investigación y Diagnóstico en Educación \\ vanesa.lancha@ehu.eus
}

Recepción: 27 de junio de 2019

Aceptación definitiva: 21 de febrero de 2020

RESUMEN: Esta investigación pretende conocer las experiencias de los profesionales de la educación con respecto a la expresión sexual del alumnado con TEA e identificar las dificultades o necesidades que puedan generarse en el proceso de intervención educativa sexual. Mediante una metodología interpretativa-descriptiva se analizan cinco dimensiones (sexualidad, manejo privacidad, interacción social, actuación profesionales y familias) del diseño generadas a través de las entrevistas realizadas a 31 profesionales. Los resultados muestran que el alumnado con TEA presenta dificultades en el manejo de habilidades sociales, una escasa conciencia de la privacidad y escaso autocontrol. Se confirma la necesidad de realizar adaptaciones personalizadas donde se atiendan necesidades e intereses específicos, trabajando y dando prioridad a las áreas relacionadas con la sexualidad ya que el alumnado muestra interés por el desarrollo de diferentes aspectos de la sexualidad y necesidad de diferentes formas de experimentarla. Los profesionales reclaman la participación activa de la familia, aunque a ambos les resulta complicado abordarlo dado que predominan tabúes, miedos y desconocimiento al respecto. Se evidencia la necesidad de desarrollar programas específicos de formación para el profesorado y la construcción de una red específica de apoyo a los profesionales para intervenir con alumnado con TEA y su entorno familiar.

Palabras clave: sexualidad; trastorno del espectro autista (TEA); educación sexual (ES); expresión sexual. 
AвSTRACT: This research aims to know about the experiences of education professionals regarding the sexual expression of students with ASD and to identify the difficulties or needs that may arise in the process of sexual education intervention. Through an interpretative-descriptive methodology, five dimensions (sexuality, privacy management, social interaction, professional and families performance) of the design are analyzed generated via interviews with 31 professionals. The results show that students with ASD have difficulties in managing social skills, little awareness of privacy and little self-control. The study confirms the need for personalized adaptations, which address specific needs and interests by working on areas related to sexuality. The students show interest in the development of aspects of sexuality and the need for different ways to experience it. The professionals claim that the active participation of the family is important. However, both professionals and families find it complicated to address the topic, due to still existing taboos, the fears and the lack of information. The need to develop specific programmes for teacher training and to construct a specific supporting network for professionals to intervene directly with students with ASD and their family environment is evident.

KEY wORDs: sexuality; autism spectrum disorder (ASD); sexual education (SE); sexual Expression.

\section{Introducción}

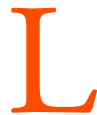

A SEXUALIDAD ES UN COMPONENTE FUNDAMENTAL DEL SER HUMANO, que abarca aspectos relacionados con el sexo, la reproducción, la intimidad, el placer y el erotismo y, también, los papeles de género, la orientación sexual y las identidades. Estos aspectos repercuten en nuestros sentimientos, acciones e interacciones y, por tanto, influyen en nuestra salud física y mental (WAS, 2015). Asimismo, es comprendida como el modo en que cada persona vive, asume, potencia y cultiva el hecho de ser sexuado, por lo que se trata de una cualidad humana evolutiva, siendo fruto del rodaje y del vivir (Amezúa, 1979).

La OMS (2006) propuso orientar la necesidad de atender y educar en sexualidad, $y$, para eso, fue de vital importancia reconocer los derechos sexuales, como el derecho a la libertad y autonomía sexual; a la equidad, la igualdad y a vivir libre de toda discriminación y violencia sexual; a la intimidad y a la privacidad sexual; a la expresión de la sexualidad, al disfrute y al placer sexual; a la elección de la pareja; a la libertad reproductiva; a la información y a la educación sexual (ES) comprensiva, y a la participación en políticas públicas sobre sexualidad (Benavides, 2017; UNESCO, 2014). Es indispensable que dichos derechos sean reconocidos, promovidos, respetados y defendidos puesto que afectan directamente al bienestar individual, interpersonal y social.

La sexualidad está estrechamente ligada al desarrollo de la persona debido a que el conocimiento sexual implica el conocimiento de sí misma o de sí mismo, el conocimiento de las demás personas y las relaciones interpersonales en un círculo social establecido (Barragán, 1996). En esta línea, no se debe eludir que en el desarrollo de la sexualidad la persona adquiere una construcción y una identidad (Barragán, Bredy y 
Rivero, 1993) a partir de la correlación entre diversos factores biológicos, psicológicos, sociales, económicos, políticos, culturales, éticos, legales, religiosos y espirituales (López, 2015).

Teniendo presente lo citado, resulta imprescindible entender la sexualidad como la manera que cada persona tiene de vivir su propio sexo, más allá de la mera genitalidad (Pellejero y Torres, 2011), ya que se trata de un aspecto fundamental en el desarrollo integral de cualquier persona pudiendo afectar directa e indirectamente a su calidad de vida y a su relación con el entorno. Por ello, en el tratamiento de términos como la sexualidad, es preciso hablar primeramente de sexualidades, pluralizar y pensar que no hay una única forma de expresión sexual, además de que la sexualidad no es un aspecto en el que todas las personas respondan a un determinado modelo, ni existe uniformidad de criterio ni de concepción (Rubin, 1984).

Debido a la importancia que tiene el desarrollo de la sexualidad, la ONU reconoce el derecho de todas las personas al acceso a una ES de calidad (Gayá, 2014). Conscientes de que las personas no nacen educadas, son los agentes educativos quienes deberán educar a lo largo de toda la vida y no únicamente durante el periodo escolar (Padrón, Fernández, Infante y París, 2009). Pese a existir un acuerdo común en cuanto a la necesidad de una ES (Oliva, 2012), se evidencia una escasa atención por el desarrollo de la ES en las distintas leyes del sistema educativo español ${ }^{1}$, además de una carencia de políticas educativas encaminadas a la ES en el sistema educativo formal (Venegas, 2017). Muchos de los conocimientos relacionados con el desarrollo de la sexualidad que obtiene el alumnado, e incluso los profesionales de la educación, provienen de cursos reducidos y privados. Además, los profesionales de la educación intervienen de manera esporádica en el aula para afrontar temáticas relacionadas con el desarrollo sexual, donde, por escasez de tiempo y espacio, tienden a ser más asistenciales que educativos. Se aborda desde la prevención y en situaciones de emergencia olvidando su valor de riqueza personal (Bejarano y García, 2016).

En este sentido, según Amezúa (2008), la ES se debe entender como un proceso abierto para el acompañamiento, donde no solo se atenderán los peligros y problemas, sino también los fenómenos y sus posibilidades. Se debe abordar desde las bases generales y no desde los problemas ocasionados por la ausencia de esas bases. Es decir, la finalidad de la ES deberá ser conseguir personas libres y responsables con su propia sexualidad y respetuosa con la de los demás; debe velar por que la persona pueda lograr la identificación e identidad sexual y generar sus propios valores y actitudes para poder vivir la sexualidad de una manera positiva, consciente y responsable dentro de su cultura. Desde un enfoque crítico y constructivista Barragán (1991) define la ES como:

... el proceso de construcción de un modelo de representación y explicación de la sexualidad humana acorde con nuestras potencialidades, con el único límite de respetar la

Con la Ley Orgánica 2/2006 (LOE), de 3 de mayo, de Educación, se apostó por la transversalidad y por incluir contenidos directamente relacionados con la sexualidad en asignaturas como Educación para la Ciudadanía y los Derechos Humanos. Sin embargo, la Ley Orgánica 8/2013 (LOMCE), de 9 de diciembre, ha preferido alejarla de las aulas. 
libertad de las demás personas, y en este sentido es necesario analizar críticamente los fundamentos de los modelos que nos proponen, contrastar diversos modelos, conocer otras culturas y la propia historia del conocimiento sexual (p. 135).

Por lo que para trabajar la ES será necesario ofrecer conocimientos que formen a las personas educandas partiendo de una educación integral, trabajando el desarrollo de valores y actitudes de respeto, igualdad, responsabilidad, libertad, autonomía, comunicación, solidaridad e inclusión y acompañar dicha ES por lo menos a lo largo de las tres etapas educativas obligatorias (Educación Infantil, Primaria y Secundaria), además de enfocarla desde un punto de vista coeducativo e integrador donde se haga partícipes a diversos agentes como la familia, el profesorado, la administración educativa, el equipo directivo, los colectivos sociales y los profesionales de la salud (De la Cruz, González, Pereyra y Santamaría, 2017; González, 2015; López, 2015).

Sin embargo, en muchos casos se da comienzo a la ES en la adolescencia, se interviene tarde y no se aborda en el momento oportuno el desarrollo de valores, actitudes y capacidades necesarias para hacer frente a una etapa de cambios continuos como es la adolescencia, sobre todo en el caso de las personas con diversidad funcional y aquellas con mayor necesidad de apoyo (Catalán y Font, 2000; López, 1990).

Las personas con mayor necesidad de apoyo necesitan, en mayor medida, aprender a conocerse, aceptarse y poder expresar su erótica de manera positiva. Así lo constata la organización Plena inclusión (2017), la cual aboga por atender, educar y prestar apoyos a la sexualidad de estas personas a través de información y ES adaptada donde se reitera la necesidad de ofrecer intimidad para que aprendan a gestionar sus estímulos eróticos, además de ofrecer autonomía para fomentar su capacidad de toma de decisiones.

Por otra parte, las relaciones interpersonales pueden jugar un papel importante en el proceso de inclusión y, para ello, será preciso ofrecer los apoyos necesarios que posibiliten poder llevar a cabo la expresión de la sexualidad. Las personas con necesidades educativas especiales, como puede ser el caso de las personas con TEA, independientemente de la severidad de sus dificultades y sus necesidades de apoyo, tienen derecho a una ES y adaptada (Koller, 2000).

La definición del TEA ha ido variando a lo largo de los años y en este estudio es asumido como una disfunción neurológica, en la que los síntomas están presentes desde la infancia temprana, aunque pueden no manifestarse plenamente hasta etapas posteriores (Gómez-León, 2019; Moro, Jenaro y Solano, 2015). Si bien la condición de las personas con TEA es compleja y variada, según el DSM-V principalmente muestran alteraciones en el desarrollo comunicativo, cognitivo y social (American Psychiatric Association, 2014). Por ello, será una prioridad trabajar en profundidad estos aspectos para que, en la medida de lo posible, puedan satisfacer sus necesidades de manera inclusiva (Travers y Tincani, 2010).

En cuanto a los aspectos comunicativos y de lenguaje, cabe destacar que las personas con TEA difícilmente podrán desarrollar conversaciones de manera recíproca y flexible. Dado que pocas personas logran comunicarse oralmente con total fluidez, es habitual hacer uso de técnicas complementarias de comunicación como pictogramas, señas o ruidos para, a través de las mismas, posibilitar la socialización de una manera 
autónoma y ayudar en la dificultad de iniciar o responder a las interacciones sociales (American Psychiatric Association, 2014). Es más, mayormente, procesan mejor la información visual que la auditiva y, por ello, para el acceso a la información es frecuente hacer uso de un lenguaje de vía visual (Mira y Grau, 2017).

En lo que respecta al desarrollo cognitivo, las áreas más afectadas son la imaginación y la capacidad de simbolización. Muchos no presentan juego simbólico y sí patrones de juego muy repetitivos y patrones de comportamiento ritualizados. La capacidad para comprender intenciones y emociones es muy limitada, reclamando habitualmente ambientes altamente estructurados y predecibles (De los Reyes, Moreno y Aguilera, 2007; Lassalle et al., 2018).

Las alteraciones antedichas generan dificultades para comprender ciertas situaciones sociales, lo que les puede llevar a mostrar comportamientos socialmente poco apropiados (Grau, Fernández y Cuesta, 2015). Así pues, en el desarrollo social muestran dificultades para adaptarse socialmente (Garrido, García, García y Carballo, 2017) y también para poner en práctica las normas sociales. Los obstáculos y dificultades que muestran para desarrollar, mantener y comprender las relaciones sociales pueden provocar sentimientos de soledad y exclusión social, que repercuten negativamente en la calidad de vida de los mismos (Baixauli, Berenguer, Colomer y Grau, 2017) y, a su vez, en su sexualidad (Gómez, Guerrero y Leiva, 2017).

Asimismo, en la sociedad actual sigue predominando el pensamiento de un único modelo social, en el que la sexualidad es un problema no resuelto en el tratamiento de las personas con TEA, ya que no persiguen el mismo modelo social y, en consecuencia, se promueve una supresión de su sexualidad, una vida solitaria y la ocultación de la actividad sexual. Actitudes como la infantilización, transmitir el enfoque en sus limitaciones y el énfasis en las mismas ante las potencialidades de la persona contribuyen hacia un panorama preocupante de invisibilidad (Dantas, Silva y Carvalho, 2014). La sexualidad de las personas con diversidad funcional está sujeta a miedos, ignorancias ${ }^{2}$ y creencias infundadas que pueden afectar tanto a las personas con TEA como a las familias y a los profesionales a la hora de iniciar el proceso de intervención educativa sexual (Alonso, 2009).

Las personas con TEA, con frecuencia, han sido consideradas sexualmente inmaduras (Ludlow, 1991), asexuadas (Zigler y Hodapp, 1986) e incluso propensas a participar en comportamientos sexuales ofensivos (Sevlever, Roth y Gillis, 2013; Stokes, Newton y Kaur, 2007). En un estudio llevado a cabo por Fernándes et al. (2016), se comprobó que la mayoría de las personas con TEA presentaban un interés por el desarrollo de diferentes aspectos de la sexualidad, aunque la cuarta parte de los participantes mostraba expresiones sexuales socialmente poco apropiadas. Según Ottoni y Maia (2019) en dichas expresiones de desventaja social están involucrados aspectos económicos, culturales, sociales, de género y de derecho.

Estos comportamientos pueden deberse a que las personas con TEA presentan una escasa conciencia de la privacidad o un conocimiento más reducido de las normas

2 Walters et al. (2013) identifican aspectos de la sexualidad como la masturbación o la menstruación, aclarando que estas pueden plantear graves riesgos para la salud y la seguridad si no son reconocidos y tratados. 
sociales (Kalyva, 2010) y, por tanto, una mayor facilidad para exteriorizar sus deseos e intereses (Haracopos y Pedersen, 1992). De igual modo, Hannah y Stagg (2016), en un análisis comparativo, indican que las personas con TEA presentan una menor conciencia sexual, menor autocontrol y menor asertividad, además de sentimientos más negativos sobre su sexualidad y su ES que las personas con un desarrollo normotípico. Respecto a las conductas sexuales, varios estudios indican que las personas con TEA participan mayormente en conductas sexuales solitarias donde se observa que las habilidades sociosexuales pueden verse afectadas en aspectos de higiene personal y toques públicos inadecuados (Gilmour, Schalamon y Smith, 2012). Por otra parte, Ottoni y Maia (2019) indican que, en ocasiones, se aborda el desarrollo sexual de los educandos como si fuera un aprendizaje "natural” y espontáneo, sin embargo, teniendo en cuenta las dificultades que presentan las personas con TEA este tipo de educación sexual informal no es eficaz.

Hay que tener presente que las personas con TEA carecen de herramientas y estrategias para autoprotegerse, por lo que los profesionales, e incluso las familias, inconscientemente, pueden caer en la tentación de prestar mayor atención a los riesgos que conlleva el desarrollo de la sexualidad generando temores, y olvidando que también son seres sexuados (Jiménez y Gutiérrez, 2006). De acuerdo con esto, los mayores miedos que reconocen las familias de los niños y niñas con TEA en el desarrollo sexual son la victimización sexual y las percepciones erróneas relacionadas con la intención de los comportamientos o expresiones sexuales de sus hijos e hijas (Ballan, 2012; Mehzabin y Stokes, 2011; Newport y Newport, 2002; Sevlever et al., 2013). Se debe contemplar una educación temprana, adaptada y completa para poder desafiar este tipo de cuestiones (Cabarcos, 2017), y en este proceso se debe poner especial énfasis a la construcción de nuevas estrategias inclusivas.

Ante esta situación, los profesionales tratan de mantener una actitud positiva y ofrecer apoyo continuo y orientación. Sin embargo, pocos profesionales se ven capacitados para presentar descripciones y análisis de los problemas sexuales además de ser escasa la experiencia de los profesionales en el ámbito de la ES de las personas con TEA (Haracopos y Pedersen, 1992). Por lo tanto, dadas las características específicas de las personas con TEA, son necesarias nuevas claves para abordar la sexualidad a través de una ES adaptada (Pask, Hughes y Sutton, 2016) puesto que la manera de vivir la sexualidad puede ser muy diferente dependiendo de la persona e incluso del grado de TEA que padezca (Dewinter, De Graff y Begeer, 2017).

Los procesos de intervención educativo-sexuales pueden ser largos y complejos (Holmes, Himle y Strassberg, 2016) y, por tanto, los agentes educativos pueden encontrarse con barreras en su camino (García-Cuevas y Hernández, 2016). Siguiendo un modelo multidisciplinar en el que se encuentren involucrados además de profesionales y especialistas, las familias, el entorno educativo y la comunidad los resultados pueden ser más efectivos y adaptativos (Mulas et al., 2010). A modo de ejemplo, en relación a la expresión sexual, se les debe enseñar a diferenciar y utilizar de manera adecuada entornos públicos y privados ofreciendo espacios y momentos para la intimidad y desarrollando, previamente, aspectos como la autonomía personal y la independencia funcional (Rodríguez, White, Arnold y Redman, 2006), sin olvidar 
proporcionar un entorno que elimine las distracciones e informar sobre los estímulos a atender (Lozano, Alcaraz y Bernabeu, 2012). Además, dadas las necesidades particulares detectadas en las personas con TEA se les debe formar en habilidades sociales desde una edad temprana como factor protector, de lo contrario, no estarán capacitadas para participar en interacciones sociales en etapas posteriores de manera coherente y efectiva, ya sea en situaciones afectivas o en contextos de amistad (Newport y Newport, 2002).

Resulta imprescindible que los profesionales e incluso las familias reciban y dispongan de una formación continua respecto a la temática (Amer, 2017), pudiendo lograr una mayor formación para hacer frente a ciertos desafíos educativos como puede ser la ES de las personas con TEA. Una adecuada información y educación redundará en un mayor aprendizaje que tendrá como consecuencia un menor grado de estrés e insatisfacción en las personas con TEA (Hatton y Tector, 2010).

A su vez, es conveniente desarrollar investigaciones que posibiliten fomentar metodologías didácticas más efectivas teniendo presentes las especificidades de las personas con TEA, pues resulta inevitable pensar que puedan tener dificultades para desarrollar su sexualidad de manera autónoma, y más aún si previamente no han recibido una ES (Lozano, Castillo, García y Motos, 2013).

Partiendo de estas premisas se ha planteado el presente estudio que pretende conocer las experiencias de los profesionales de la educación con respecto a la expresión sexual del alumnado con TEA e identificar dificultades o necesidades que puedan generarse en el proceso de intervención educativa sexual.

\section{Metodología}

El presente estudio, exploratorio, se ha desarrollado haciendo uso de una metodología cualitativa, que ha permitido realizar un análisis concreto de casos singulares. Se han recogido opiniones, sentimientos y posibles propuestas educativas sexuales de los participantes.

\subsection{Participantes}

En el estudio han participado 31 profesionales (27 eran mujeres y 4 hombres) de centros educativos integrados en la asociación Gautena ${ }^{3}$, que trabajan con alumnado con TEA. Sus edades están comprendidas entre los 23 y 56 años y corresponden a un centro de Educación Infantil, dos de Primaria y cuatro de Secundaria. Pertenecen a estratos profesionales diferentes: profesorado $(n=7)$, educadoras $(n=8)$ y cuidadoras $(n=16)$.

En la selección de los participantes se ha tenido en cuenta que fueran profesionales de amplia experiencia (superior a 3 años) y que representaran a todo el espectro de

La Asociación Guipuzcoana de Autismo, Gautena, es una entidad sin ánimo de lucro, cuyo objetivo es informar y sensibilizar a la sociedad sobre la problemática que supone el autismo y ofrecer los servicios adecuados a las personas que presentan este tipo de trastorno. 
profesionales que trabaja con el alumnado para posibilitar que las conclusiones del estudio tuvieran la necesaria validez externa. Los participantes provienen de aulas estables de Gipuzkoa ${ }^{4}$, y constituyen una muestra representativa de los profesionales del servicio educativo de la provincia ${ }^{5}$ y, habida cuenta de los objetivos del estudio, su representatividad se puede ampliar al Estado español.

Asimismo, dado que el colectivo de alumnos y alumnas con TEA se caracteriza principalmente por la dificultad de desarrollar un lenguaje oral fluido y por la dificultad de desarrollar capacidades sociales, se consideró necesario recabar información de personas con TEA correspondientes a los tres niveles de apoyo en función de los déficits que muestran, aunque el efectuar un estudio comparativo de los mismos superaba los objetivos de este estudio.

\subsection{Instrumento}

Se han realizado entrevistas semiestructuradas, tanto individuales como grupales. Estas últimas se han efectuado con dinámicas de grupos de discusión. Tras llevar a cabo entrevistas individualizadas, dado que la temática a abordar resultaba compleja y personal para algunos de los profesionales, se constató la necesidad de complementar la información recogida mediante 7 dinámicas de grupos de discusión, desarrolladas en aulas estables.

El diseño básico de la entrevista consta de 19 preguntas abiertas relacionadas con la ES de las personas con TEA y las experiencias de los profesionales en este proceso (ver Tabla 1).

\begin{tabular}{|c|c|}
\hline \multicolumn{2}{|c|}{$\begin{array}{l}\text { TABLA 1. Dimensiones derivadas de las respuestas de las entrevistas } \\
\text { individuales y grupales }\end{array}$} \\
\hline \multicolumn{2}{|r|}{ EDUCACIÓN SEXUAL (ES) } \\
\hline Sexualidad & $\begin{array}{l}\text { - Qué se entiende por sexualidad } \\
\text { - La importancia que se le otorga al desarrollo de aspectos } \\
\text { de la sexualidad }\end{array}$ \\
\hline Manejo de la privacidad & $\begin{array}{l}\text { - La importancia de favorecer espacios diversos } \\
\text { - Comprensión de las normas sociales } \\
\text { - Complicaciones adaptativas en entornos comunes } \\
\text { - Habilidad social y autocontrol en el espectro autista }\end{array}$ \\
\hline Interacción social & $\begin{array}{l}\text { - Conductas interpersonales con agentes internos (aula esta- } \\
\text { ble) y agentes externos (entorno social) }\end{array}$ \\
\hline
\end{tabular}

4 En la actualidad, encontramos 19 aulas estables en Gipuzkoa donde se integra a una diversidad de alumnado con TEA y alumnado con altas necesidades de apoyo entre 3 y 21 años.

5 Las aulas estables del servicio educativo de Gautena cuentan con 87 profesionales entre maestros, educadores, especialistas de apoyo educativo y cuidadores. 


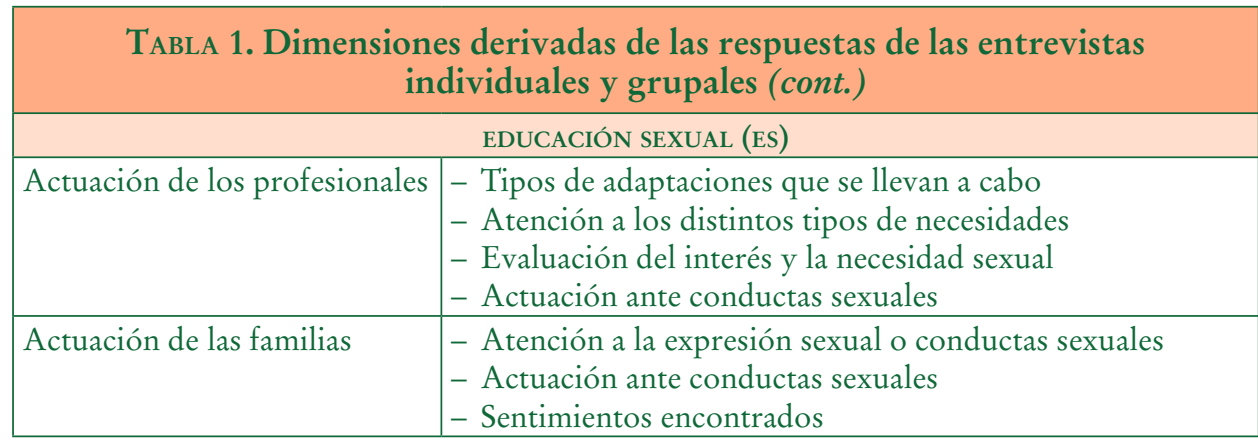

\subsection{Procedimiento}

Las entrevistas al profesorado se realizaron en los propios centros y, en todos los casos, fueron responsables de alumnos y alumnas con TEA. Con el permiso de la dirección del servicio educativo se les informó a todos los profesionales sobre la intención del proyecto. Una vez garantizados el anonimato y la confidencialidad de la información proporcionada los participantes dieron su consentimiento.

Con carácter previo a la realización de las entrevistas se vio la necesidad de concretar el concepto de sexualidad que se asumió en el proceso de recogida de datos. Así pues, se ha entendido la sexualidad como un componente fundamental que abarca aspectos relacionados con el sexo, la reproducción, la intimidad, el placer, el erotismo, las identidades, entre otros. Además, se ha considerado la ES como un derecho necesario a través del cual se debe conseguir personas libres y responsables con su propia sexualidad.

A pesar del enfoque ofrecido en el desarrollo del instrumento se ha priorizado perseguir las propias percepciones y opiniones de los participantes con intención de poder realizar un análisis de la realidad educativa sexual de las personas con TEA.

\section{Resultados}

Los resultados obtenidos se presentan siguiendo el esquema de las cinco dimensiones planteadas en el diseño que, a su vez, conforman el objeto de estudio: sexualidad, manejo de la privacidad, interacción social, actuación de los profesionales y de las familias.

\subsection{Sexualidad}

Se ha percibido la existencia de dos grupos a la hora de comprender la sexualidad. Por un lado, varios profesionales ofrecen una perspectiva genitalizada donde consideran que la sexualidad abarca únicamente aspectos como el placer sexual y la erótica: 
"Para mí la sexualidad es el conjunto de actividades y comportamientos relacionados con el placer sexual” (MESP05) ${ }^{6}$. Consideran que la sexualidad se magnifica o se vive con mayor intensidad en la adolescencia, omitiendo la importancia del desarrollo sexual en otras etapas educativas como la etapa infantil, la adulta o la vejez.

El segundo grupo de profesionales, que son la mayoría, consideran que la sexualidad abarca, además de aspectos físicos y fisiológicos, aspectos psíquicos y mentales. Defienden que la sexualidad no empieza ni termina en los órganos sexuales de las personas y comprenden la sexualidad más allá de la mera genitalidad, integrando aspectos psicológicos y sociales. Asimismo, subrayan la necesidad de abordar temáticas relacionadas con la sexualidad en todas las etapas evolutivas y, para ello, será preciso poseer unos mínimos conocimientos al respecto: “La sexualidad está en nuestro día a día sin que siquiera nos demos cuenta, en nuestras emociones, pensamientos, relaciones sociales..." (HESP17). Es por eso que insisten en darle la misma consideración al desarrollo de la sexualidad en todas las edades y no únicamente en la adolescencia, aunque en esta etapa vital pudiera existir mayor confusión a causa de los cambios hormonales: "En la adolescencia existe más necesidad de explorar el cuerpo y conocerse, ir viendo cómo me sitúo en este mundo” (MESP21). Indican que el deseo sexual y el deseo por explorarse a sí mismos no desaparece una vez transcurrida la adolescencia, sino que pueden llegar a regularse por sí solos y dirigirlos a momentos oportunos.

Subrayan la necesidad de empezar a trabajar la ES en etapas tempranas, puesto que no se trata únicamente de intervenir cuando el deseo sea visible, sino de conocer la anatomía y fisiología de los genitales, los cambios físicos a lo largo del desarrollo, la relación con el propio cuerpo, las diferencias entre sexos, el respeto hacia las demás personas y sus deseos, así como expresar sentimientos, manifestaciones afectivas: "Cuanto antes se trate, más fácil resultará gestionar comportamientos y conductas sexuales” (MESE02). Destacan que la mayoría del alumnado se ve capacitado para reconocer las partes del cuerpo y las diferencias entre sexos, pero detectan conocimientos escasos sobre el placer corporal y las prácticas sexuales, además de poca fluidez en las relaciones interpersonales.

Estos profesionales recalcan que abordar temáticas relacionadas con la sexualidad resulta imprescindible para el desarrollo personal: "La sexualidad forma parte de la expresión del ser humano, por lo que lo vemos muy necesario” (MESP01). Observan que cada persona tiene su propia manera de vivir su sexualidad además de que el alumnado con TEA, continuamente, trata de explorar su propio cuerpo, mostrando curiosidad por desarrollar aspectos relacionados con la sexualidad. Sin embargo, cuando una acción les resulta placentera, puede llegar a formar parte de su rutina diaria, haciendo caso omiso de su entorno.

6 La codificación utilizada sigue los siguientes criterios:

1. ${ }^{\mathrm{a}}$ letra: M (Mujer), H (Hombre)

2. ${ }^{\mathrm{a}}$ y 3. ${ }^{\mathrm{a}}$ letra: EI (Educación Infantil), EP (Educación Primaria), S (Educación Secundaria)

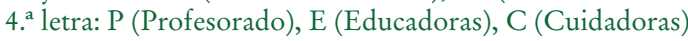

2 cifras: Código en cada estrato 


\subsection{Manejo de la privacidad}

Los profesionales son conscientes de las dificultades que presentan las personas con TEA para comprender las normas sociales y el concepto de la intimidad y, por consiguiente, concluyen que difícilmente tienen en cuenta a la otra persona y que difícilmente comprenden cuándo una acción está bien o está mal. Consideran que no tienen conciencia absoluta de la mirada de los demás y, por tanto, son capaces de llevar a cabo acciones que puedan resultar socialmente poco apropiadas. Uno de los profesionales, en referencia a los límites de una persona con TEA, apuntó que: "Si yo tengo una sensación, me he descubierto, me toco, me gusta y sigo. Si en ese momento no hay una intervención, una regulación de un adulto que le enseñe dónde, cuándo y cómo se debe desahogar, si eso no existe desde un primer momento, ellos siguen utilizando la misma pauta, porque no son capaces de regularse por sí solos” (MEIE10). Observan dificultades en el manejo de las habilidades sociales, una escasa conciencia de la privacidad y escaso autocontrol, por lo que, en muchas ocasiones, entran en conflicto a la hora de comprender por sí solos la visión de los demás.

Ante el alumnado que no sabe regular este tipo de intereses, los profesionales ven la necesidad de ofrecer un espacio para que puedan relajarse. No obstante, aclaran que los colegios no tienen por qué ofrecer espacios para favorecer la intimidad, ya que no consideran que sea un espacio adecuado. Opinan que el colegio es una institución para enseñar y aprender, de modo que el entorno ideal para trabajar ciertos aspectos de la sexualidad de la persona es el hogar, un entorno donde se pueda ofrecer intimidad. Los participantes apoyan trabajar la ES con el alumnado dentro del colegio, pero no practicarla.

En casos en los que el alumnado con TEA se encuentre impaciente y nervioso, los profesionales apuntan que pueden llegar a hacer uso de los baños públicos del colegio para que el alumno o alumna se pueda relajar, siempre y cuando previamente haya recibido unas pautas básicas: "La persona puede haber manifestado una conducta sexual inadecuada en un lugar público, aunque no fuera el momento oportuno porque podría no ser consciente de sus actos y no saber cómo gestionar dicha conducta" (MESC03). No creen que sea deber de los profesionales de la educación, por ejemplo, enseñar a su alumnado a masturbarse, ya que tareas como estas deberían estar en manos de las familias o profesionales especializados.

En los casos en los que se les permita masturbarse en el baño del colegio, matizan que se les debe aclarar que ese no será siempre su espacio para masturbarse: "Educar desde el principio, no a no hacer, si no a cómo, cuándo y dónde” (MESP21). A pesar de las posibles alternativas ante tal situación hay aspectos del desarrollo personal que forman parte de otro entorno, un entorno más privado e íntimo, el hogar. Consideran que la prioridad como profesionales debería ir encaminada a potenciar otros aspectos del desarrollo personal.

Por otro lado, los participantes consideran que la utilidad de las normas sociales es necesaria para que todos y todas podamos regular nuestra conducta. Sin embargo, el alumnado con TEA, inicialmente, no llega a comprender el sentido de esas normas, de ahí la importancia de enseñar y trabajar dichas normas sociales desde etapas 
tempranas. Perciben que ante una necesidad como esta no tienen en cuenta dónde y con quién están en ese momento. Por eso, como bien se ha mencionado en anteriores dimensiones, observan que, en ocasiones, suelen llevar a cabo comportamientos socialmente poco apropiados, como desnudarse en la calle, abrazar o besar a un desconocido. En estos casos, el criterio deberá ser el mismo, siempre teniendo en cuenta que tienen mayor dificultad para comprender las normas sociales y para regularse por sí solos: "El mensaje debe ser muy claro, cuanto más difícil lo tienen más claro tiene que ser el mensaje, no puede haber ambigüedades, no puede dudar de si es una norma o no es una norma” (MESC27). En el colegio, actividades como gimnasia, piscina y musicoterapia son más propicias para que surjan conductas sexuales. En este tipo de entornos muchos profesionales han podido identificar algunos fetiches de su alumnado debido a que no existen mesas ni una estructura regulada, por ello, es necesario aclarar qué son las normas sociales y cómo y cuándo se deben respetar.

En este aspecto, los profesionales se ven con dificultades para gestionar ciertas conductas de su alumnado, ya que piensan que prohibir no es la mejor opción. No obstante, en muchas ocasiones tienden a la prohibición o a interrumpir con rapidez por no propiciar una situación desagradable en un entorno social. A la hora de tratar de desviar una conducta que no es adecuada, el alumnado con TEA suele mostrar nerviosismo y, por ello, se requiere regular este tipo de conductas en la etapa infantil y no esperar a que se incrementen en etapas posteriores: "En la infancia no llaman tanto la atención, pero en etapas posteriores son conductas intolerables” (MEPC15). Matizan que no se trata de prohibir, sino de regular dicha conducta y enseñarle cuál es el lugar más adecuado. En cualquier caso, los participantes aclaran que, si después de una prohibición no existe una explicación o unas pautas a seguir, el alumnado con TEA puede comprender el proceso de desarrollo sexual de manera equivocada, predominando en ellos sentimientos negativos.

\subsection{Interacción social}

El profesorado, debido a la posibilidad de que se generen conflictos, no propicia que el alumnado con TEA, en momentos como el recreo, interaccione con los demás alumnos y alumnas y de aquí se deriva que la relación e interacción con el resto del alumnado sea escasa, lo que dificulta la plena inclusión. En muchos casos, para que se relacionen entre ellos, el adulto tiene que ayudar a establecer las relaciones. Además, algunos de los profesionales consideran que el alumnado con TEA normalmente solo es aceptado por su círculo familiar y los profesionales que los rodean: "El resto de alumnos a veces lo pasan mal, no saben cómo relacionarse con nuestros alumnos, les cuesta” (HESC28).

Asimismo, a muchos de los estudiantes con TEA les resulta complicado diferenciar las actividades que pueden realizar tanto con una persona conocida como con una desconocida, por lo que, en ocasiones, desarrollan acciones que quizás no sean las más acertadas como, por ejemplo, rozar u oler a una persona desconocida o agarrar de las manos y pedir saltar a una persona desconocida: "Hay conductas que no son nada 
apropiadas por la gente del exterior y por la edad que tienen, por eso hay que empezar a actuar enseguida" (MEPC25).

A pesar de este tipo de controversias, los profesionales indican que no suelen recibir ninguna queja por parte de agentes externos, aunque tienen claro que la perspectiva de un docente de aula ordinaria y un docente de aula estable es diferente hasta el punto en el que comprenden el desconocimiento y la distancia que muestran algunos profesionales y alumnos externos al aula. Lo aclaran diciendo que: "Hay que reconocer que nosotros ya estamos acostumbrados a este tipo de conductas, aunque a veces nos parezcan desgarradores algunos gritos ya estamos acostumbrados, y muchos profesores a veces se asustan y se alarman" (MEPC16). A veces, suelen sentirse frustrados e incomprendidos ante situaciones en las que los agentes externos no llegan a comprender la necesidad de su alumnado.

Los profesionales consideran que la aceptación de las diferencias y similitudes entre el alumnado de las aulas ordinarias y las aulas estables es reseñable. Igualmente, para que los agentes externos no tengan reparo en acercarse al alumnado del aula estable y poder entablar una relación entre ellos, cada año los profesionales del aula estable proponen una formación general en cada colegio para que los agentes externos puedan conocer con mayor profundidad lo que conlleva el autismo al mismo tiempo que la convivencia entre el alumnado y los profesionales pueda mejorar de forma progresiva.

\subsection{Actuación de los profesionales}

Siempre que resulte necesario los profesionales tratan de abordar temas relacionados con el desarrollo de la sexualidad a través de un programa individualizado adaptado (PIA), ya que suelen predominar aspectos como el trabajo autónomo, la comunicación y la comprensión. El programa individual consta de unas pautas de actuación educativas específicas elaboradas para cada persona, y responde a aspectos relacionados con la autonomía personal, con las relaciones sociales, con aspectos comunicativos y otras dimensiones evolutivas. Les resulta necesario tener presentes las características singulares de cada alumnado para poder ofrecer una ES adaptada, de manera que se aborde la ES en función de las características de la persona: "Los programas de ES no deben nunca generalizar sus teorías sobre un colectivo... tienen que ser los programas los que se ajusten a cualquier tipo de demanda que surja” (MEPP23).

Por lo tanto, asumen la responsabilidad de valorar y trabajar cuando la necesidad y el deseo sexual son visibles incorporando objetivos concretos en el PIA. En el caso de que el alumnado no muestre indicios de un interés por desarrollar aspectos relacionados con la sexualidad, normalmente, no suelen prestar especial atención ya que predominan otros aspectos de mayor importancia en la vida diaria, como son la autonomía personal y la comunicación. Entre los profesionales se percibe la existencia del temor a que por el simple hecho de iniciar una ES se despierte el deseo sexual, e incluso a que las personas externas perciban el comportamiento sexual de las personas con TEA de forma equivocada. 
Los participantes afirman que tratan de comprender qué hay detrás de cada comportamiento sexual y, para ello, no solo miran el comportamiento puntual, sino que tratan de analizar el origen del mismo. Tienen en cuenta que las razones de este tipo de conductas pueden ser muy variadas, como pueden ser querer masturbarse, tener una alergia que produzca picores, querer llamar la atención, tener una incomodidad física o razones médicas: "Nosotras sobre todo intentamos buscarles una explicación a los problemas de conducta, cuando a su vez también se manifiestan comportamientos sexuales... no hay que ir a la conducta sino al origen de la conducta" (MESC07). Apuntan que en la mayoría de casos los problemas de conducta suelen darse por falta de comunicación e incomprensión.

Ante este tipo de situaciones consideran necesaria la información previa del alumnado. Es decir, toda información puede ser relevante para la posible actuación de los profesionales, por ello, es importante que los profesionales y las familias se coordinen y cooperen cada uno a su nivel. Indican que comprender las características y necesidades de cada alumno y alumna es importante para poder actuar y ofrecer una respuesta adecuada. Tienden a combinar la información de las familias con las observaciones de anteriores profesionales o instituciones a través de un cuestionario de visión personal. Estiman importante obtener diferentes puntos de vista ya que en ocasiones ha sucedido que la información recibida por parte de la familia resulta escasa, bien porque ciertos comportamientos los puedan llegar a considerar como manías o hábitos o, simplemente, porque no han considerado que fueran conductas relevantes como, por ejemplo, realizar frotamientos contra la mesa o la pared, prestar especial atención a colores u olores vivos, quitar y ponerse la ropa continuamente en entornos sociales: "Es importante en cualquier acogida informar de todo lo que sea posible sobre cada individuo, ya que cualquier información puede ser relevante para los profesionales, para que estos puedan darle el trato, la educación y el entorno que mejor le conviene" (HESE18).

A partir de aquí los profesionales comienzan a valorar y trabajar estrategias posibles para gestionar y regular la conducta del alumnado. Sugieren que tratan de controlar o resolver de manera prudente este tipo de situaciones a pesar de que, en ocasiones, no se sienten capacitados para responder a este tipo de desafíos, porque dudan de cuál podría ser la mejor respuesta, pues consideran que prohibir o suspender la actividad puede no ser la mejor opción. Insinúan la necesidad de formarse para poder abordar un tema que consideran personal y complejo. A este respecto manifiestan que "seguimos teniendo la necesidad de tratar la ES y las conductas sexuales con la dedicación y la atención que requiere" (MESC04).

Además, evidencian la necesidad de normalizar el entorno del alumnado en todo momento: "Lo principal es asumir y después normalizar, ya que si no normalizamos la situación nunca podrá tener una educación sana” (MESC19). De esta forma, consiguen que el alumnado integre el aprendizaje adquirido en su vida diaria para que puedan realizar las actividades de manera autónoma. Son personas que requieren de una rutina, una estructura y unas costumbres, por lo que los profesionales para ayudar a adquirir aprendizaje y conocimientos hacen uso de pictogramas, imágenes y explicaciones sencillas. 


\subsection{Actuación de las familias}

Los profesionales señalan que en este proceso la participación de la familia es imprescindible para el buen desarrollo de los objetivos acordados en la programación individualizada adaptada (PIA). Al fin y al cabo, tanto los profesionales como las familias son agentes que forman parte de la vida diaria de las personas con TEA y, en consecuencia, se reitera la necesidad de que todos los agentes educativos participen y cooperen en el aprendizaje del alumnado con TEA.

Las familias deberán ofrecer a su hijo o hija pautas o instrucciones para gestionar el desarrollo y expresión sexual de manera adecuada, además de ofrecer momentos para la intimidad. Sin embargo, son muchas las familias a las que se les hace complicado afrontar el proceso de ES y, por ello, en muchas ocasiones, los profesionales aseguran encontrarse con obstáculos ya que consideran que ciertos aspectos del desarrollo de la sexualidad de las personas con TEA deben atenderlos las familias y no los profesionales de la educación, entendiendo que deben ser espacios íntimos (hogar) los lugares donde deban satisfacer su deseo sexual y no, por ejemplo, los baños del colegio.

Los profesionales indican que en el caso de que la familia trabajara desde una edad temprana y de forma natural la sexualidad de su hijo o hija, probablemente no haría falta tener que responder a conductas socialmente poco apropiadas dentro del horario escolar, ya que dentro de su calendario diario tendría un momento para disfrutar de su intimidad y sus deseos: "Si yo reconozco que mi hijo tiene esa necesidad y lo reconozco, es que tengo que hacer algo. Y en cambio si digo bueno ya se le pasará, podemos pasar a un problema. Si hay un proceso de aprendizaje este tipo de conductas se canalizan de manera más adecuada y su sexualidad, normalmente, tampoco suele ir canalizada hacia la relación con otros, sino a sí mismos o, como mucho, a la observación de otra persona o de otro objeto” (MEIP09).

Aunque los profesionales sean conscientes de que se trata de una temática complicada para la familia, incluso un reto educativo, afirman que alguien debe tomar la responsabilidad para que la persona pueda desarrollar su sexualidad de manera autónoma. Así, indican que "el mayor problema es que en casa es un tema tabú, y cuesta trabajarlo, y si no sabe hacerlo por sí solo porque aprende sin ayuda, al final es muy difícil que el padre o la madre diga ya le voy a enseñar yo, y a nosotras es lo que nos parece más difícil. Si alguien le enseñara, y él supiera hacerlo, podría llegar a hacerlo a la mañana o a la noche en su hogar, sin necesidad de ningún espacio en el aula del colegio" (MESP04). Se percibe que el hecho de que todavía siga siendo un tema tabú puede condicionar el desarrollo sexual de las personas con TEA.

Asimismo, en el caso de que la familia no se vea capacitada para llevar a cabo esta tarea, los profesionales suelen ofrecer posibles pautas de actuación e incluso la posibilidad de redirigir esa necesidad a un centro especializado. Existe mucho desconocimiento y miedo respecto a la temática, y es por ello que ven necesaria la formación en ES para las familias de manera que no recaiga la total responsabilidad en los profesionales de la educación. 


\section{Discusión y conclusiones de los resultados}

Se ha constatado que la manera de vivir la sexualidad puede ser muy diferente dependiendo de la persona y, también, del grado de TEA que tenga (Dewinter et al., 2017). La mayoría del alumnado presenta interés por el desarrollo de diferentes aspectos de la sexualidad y, sin embargo, les resulta complicado desarrollar los mismos de manera autónoma en el caso de no haber recibido una ES previa (Lozano et al., 2013). Se han corroborado los resultados de Hannah y Stagg (2016) y Kalyva (2010), donde indican que los estudiantes con TEA presentan una escasa conciencia de la privacidad, escaso autocontrol y dificultades en el manejo de las habilidades sociales. Viven el desarrollo de su sexualidad de forma más natural, por lo que tienen mayor facilidad para exteriorizar sus deseos e intereses y, en consecuencia, los profesionales contemplan que algunos de los comportamientos de su alumnado pueden resultar socialmente poco apropiados como, por ejemplo, masturbarse en un entorno social u observar detenidamente a niños y niñas (Fernándes et al., 2016; Stokes et al., 2007). Este tipo de actividades se dan porque a las personas con TEA les resulta complicado comprender en su totalidad las normas sociales debido a las dificultades que muestran para adaptarse socialmente, y esto genera prestar mayor atención a las limitaciones olvidando sus potencialidades (Dantas et al., 2014; Garrido et al., 2017). Esta situación donde las habilidades sociosexuales se ven afectadas (Gilmour et al., 2012) puede repercutir de manera negativa en el desarrollo de su sexualidad (Gerdel et al., 2017), pudiendo generar sentimientos de soledad o exclusión social (Baixauli et al., 2017). También, se ha comprobado que en la sociedad actual todavía predomina un único modelo social, lo que condiciona el desarrollo sexual de las personas con TEA, ya que estas personas no siguen el mismo modelo y, por consiguiente, mayormente, solo son aceptadas por su círculo familiar y los profesionales que los rodean (Rubin, 1984). De modo que, dadas las características singulares de las personas con TEA en ocasiones, se encuentran con barreras en el camino de su inclusión socioeducativa (García-Cuevas y Hernández, 2016).

Por otro lado, se ha percibido una escasa atención por el desarrollo de la ES de los estudiantes (Bejarano y García, 2016) porque tanto a los profesionales como a las familias de las personas con TEA les resulta complicado trabajar este tópico y más aún cuando surgen aspectos como la masturbación (Walters et al., 2013). A este respecto, se ha constatado que uno de los mayores temores de las familias, e incluso de algunos profesionales, son las percepciones erróneas relacionadas con la intención de los comportamientos sexuales de las personas con TEA (Ballan, 2012; Mehzabin y Stokes, 2011; Newport y Newport, 2002; Sevlever et al., 2013). Los profesionales perciben el miedo y la angustia de muchas familias a la hora de abordar temas relacionados con la sexualidad debido a que, para muchas, aún se trata de un tema tabú, difícil de abordar con normalidad y naturalidad. Esto puede obstaculizar el proceso de intervención sexual, ya que es imprescindible la participación activa de las familias además de una colaboración y coordinación adecuada entre ambos (Travers y Tincani, 2010).

Según los profesionales, se corroboró que las personas con TEA pueden lograr mejores resultados si las familias están implicadas en el proceso de atención temprana (García et al., 2015). Por lo que, la familia, siendo un apoyo indispensable, será quien 
deba tomar la principal y primera responsabilidad, junto con la ayuda de los profesionales de la educación, donde juntos se encargarán de dar comienzo a la ES. Es importante reconocer y abordar las expectativas que tienen las familias y los profesionales sobre el desarrollo sexual de las personas con TEA, teniendo siempre presente que los procesos de intervención sexual con estas personas son largos y complejos (Holmes et al., 2016).

Por ello, se ha observado que la ES sigue siendo un reto educativo para muchos de los profesionales puesto que muchos de ellos han adquirido conocimientos sobre la educación sexual de forma espontánea, estrategia impensable en personas con TEA dadas las necesidades específicas que presentan, de modo que este tipo de educación informal no es eficaz para los mismos (Ottoni y Maia, 2019). Ello implica la necesidad de una formación continua, de manera que puedan atender a las dificultades que presentan las personas con TEA y generar estrategias positivas de apoyo (Amer, 2017). Si bien es cierto que los profesionales tratan de trabajar la ES de este alumnado de una manera positiva y preventiva, teniendo en cuenta las características singulares de expresión sexual que pueden generar estas personas, la respuesta más común suele ser la prohibición y, de esta manera, existe un cierto riesgo de que el alumnado comprenda el proceso de desarrollo sexual de una manera negativa (Hannah y Stagg, 2016). Ante las adversidades se deben generar oportunidades de mejora y estrategias para atender a la diversidad sexual y evitar en la medida de lo posible la prohibición (Pask et al., 2016), ya que tienen derecho a una ES independientemente de su funcionamiento intelectual o la severidad de sus dificultades (Koller, 2000).

En las estrategias educativas sexuales se deben tener en cuenta las fortalezas de la persona puesto que serán útiles para incrementar la capacidad de comprensión y adquirir nuevas habilidades. Es preciso, además de formar en habilidades sociales desde una temprana edad, organizar el entorno educativo, manejar comportamientos desadaptativos y desarrollar estrategias que faciliten el aprendizaje, sin olvidar ofrecerles espacios para el aprendizaje y para la intimidad (Newport y Newport, 2002).

A modo de conclusión, se debe enfatizar que los resultados y conclusiones obtenidas mediante la presente investigación no permiten realizar amplias generalizaciones puesto que la muestra de este estudio se ha basado en profesionales procedentes de la Asociación Guipuzcoana de Autismo, Gautena. Sin embargo, se destaca que las cuestiones relacionadas con la sexualidad de las personas con TEA son un objetivo social que no se puede eludir. Se percibe un intento de asumir el proceso de ES desde un enfoque positivo, generando un entorno carente de prejuicios y tabúes, sin embargo, para muchos de los profesionales todavía sigue siendo un reto educativo, debido a que predominan sentimientos de desconocimiento y miedo.

Es importante que los profesionales se actualicen y se formen en temáticas relacionadas con la sexualidad de las personas con TEA y, en este sentido, es necesario desarrollar programas específicos de formación para el profesorado con intención de reducir el desconocimiento sobre la ES.

Teniendo en cuenta las características específicas y la posible severidad de las dificultades o necesidades de las personas con TEA, se concluye que difícilmente puede existir un único método que sea siempre el idóneo para la intervención sexual, 
puesto que se debe adaptar a las peculiaridades individuales y al entorno sociofamiliar. Aunque los procesos de intervención sexual en personas con TEA resulten largos, complejos y exijan paciencia, todos los niños y niñas son educables. Al fin y al cabo, la intervención educativa sexual debe programarse con objeto de adquirir capacidades y habilidades nuevas, superando la mirada hacia lo que no pueden hacer y teniendo presente que son seres sexuados.

Asimismo, se hace necesaria la construcción de una red específica de apoyo al profesorado que intervenga directamente con el alumnado con TEA y la familia. Mediante esta se debe contribuir a la difusión de información actualizada y experiencias positivas entre los profesionales y las familias, que les permita superar las percepciones basadas en tabúes y prejuicios, a la vez que se generen espacios de discusión y reflexión al respecto. Por ello, para lograr unos resultados eficaces, la intervención educativa sexual debe basarse en el consenso entre la familia y los profesionales del centro educativo.

El compromiso de un cambio se establece en base a la capacitación de los profesionales, e incluso de la comunidad en general, lo que puede contribuir al aumento de la calidad de vida de las personas con TEA, por lo que el estudio de los procesos de intervención educativa y la relación con el desarrollo educativo del alumnado con TEA son una línea de investigación futura. Así pues, resulta necesaria la continuidad de este trabajo debido a las dificultades identificadas a la hora de atender a la expresión sexual de las personas con TEA. Un análisis de necesidades exhaustivo nos puede orientar en los diseños de programas específicos de educación sexual donde se incorporen aspectos como, por ejemplo, habilidades sociales, comunicación, incluso la enseñanza de la masturbación.

\section{Referencias bibliográficas}

Alonso, J. R. (2009). Autismo y síndrome de Asperger: guía para familiares, amigos y profesionales. Salamanca: Amarú.

Amer, A. A. F. (2017). The level of the use of positive behavioral support strategies by teachers of children with autism spectrum disorders in Amman. College Student Journal, 51(1), 81-90.

American Psychiatric Association (2014). Guía de consulta de los criterios diagnósticos del DSM-5. España: Editorial Médica Panamerica.

Amezúa, E. (1979). La sexología como ciencia: esbozo de un enfoque coherente del hecho sexual humano. Revista de Sexología, 1, 17-28.

Amezúa, E. (2008). Avances en educación sexual. La asignatura de los sexos. Anuario de Sexología A.E.P.S., 10, 139-154.

Baixauli, I., Berenguer, C., Colomer, C. y Grau, M. D. (2017). Intervenciones para promover la comunicación social en niños con trastornos del espectro autista. Revista de Neurología, 64(1), 38-44.

Ballan, M. (2012). Parental perspectives of communication about sexuality in families of children with autism spectrum disorders. Journal of Autism and Developmental Disorders, 42(5), 676-684. 
Barragán, F. (1991). La educación sexual. Guía teórica y práctica. Barcelona, Buenos Aires, México: Paidós.

Barragán, F. (1996). La educación sexual y la Educación Secundaria Obligatoria: enseñar a creer o aprender a crear. Materiales curriculares Educación Secundaria Obligatoria. Junta de Andalucía. Consejería de Educación y Ciencia.

Barragán, F., Bredy, C. y Rivero, Z. (1993). Programa ITAKA: Constructivismo y educación sexual para el profesorado de ciencias naturales. Qurriculum: Revista de Teoría, Investigación y Práctica Educativa, 6-7, 203-216.

Bejarano, M. T. y García, B. (2016). La educación afectivo-sexual en España. Análisis de las leyes educativas en el periodo 1990-2016. Opción [En línea], 32(13), 756-789.

Benavides, A. (2017). Bioética en sexualidad y reproducción humana. Revista Peruana de Ginecología y Obstetricia, 63(4), 565-572.

Cabarcos, J. L. (2017). Atención psicoeducativa en alumnos con TEA. Revista Padres y Maestros, 369, 20-25.

Catalán, E. y Font, P. (2000). Presente y futuro de la educación afectivo sexual. Aula de Innovación Educativa, 19, 41-44.

Dantas, T. C., Silva, J. S. S. y Carvalho, M. E. P. (2014). Entrelace entre gênero, sexualidade e deficiência: uma história feminina de rupturas e empoderamento. Revista Brasileira de Educação Especial Marília, 20(4), 555-568. https://doi.org/10.1590/S1413-65382014000400007.

De la Cruz, C., González, B., Pereyra, C. y Santamaría, C. (2017). Posicionamiento de Plena inclusión por la necesidad de educar y dar apoyo a la sexualidad de las personas con discapacidad intelectual o del desarrollo. Plena inclusión.

De los Reyes, I., Moreno, F. J. y Aguilera, A. (2007). La atención educativa en el caso del alumnado con trastornos del espectro autista. Revista de Educación, 344, 425-445.

Dewinter, J., De Graaf, H. y Begeer, S. (2017). Sexual orientation, gender identity, and romantic relationships in adolescents and adults with autism spectrum disorder. Journal of Autism and Developmental Disorders, 47(9), 2927-2934.

Fernándes, L., Gillberg, C., Ceederlund, M., Hagberg, B., Gillberg, C. y Billstedt, E. (2016). Aspects of sexuality in adolescents and adults diagnosed with autism spectrum disorders in childhood. Journal of Autism and Developmental Disorders, 46(9), 3155-3165.

García-Cuevas, A. y Hernández, M. E. (2016). El aprendizaje cooperativo como estrategia para la inclusión del alumnado con TEA/as en el aula ordinaria. Revista de Educación Inclusiva, 9(2), 18-34.

Garrido, G., García, M., García, R. y Carballo, G. (2017). Perfil comunicativo y de adaptación social en población infantil con trastorno del espectro autista: nuevo enfoque a partir de los criterios del DSM-5. Revista de Neurología, 65(2), 49-56.

GAYÁ, V. (2014). Educación sexual: asignatura pendiente. La LOMCE elimina prácticamente esta materia de las aulas. El Siglo de Europa, 1058, 46-47.

Gilmour, L., Schalomon, P. M. y Smith, V. (2012). Sexuality in a comumunity based sample of adults with autism spectrum disorder. Research in Autism Spectrum Disorders, 6, 313318. https://doi.org/10.1016/j.rasd.2011.06.003.

Gómez-León, I. (2019). A través de los ojos del niño con trastorno del espectro autista. Revista Internacional de Educación y Aprendizaje, 7(1), 11-22.

Gómez, M. A., Guerrero, J. F. y Leiva, J. J. (2017). Análisis de la inclusión socioeducativa de jóvenes con síndrome de Asperger: un estudio cualitativo. Revista Internacional de Investigación e Innovación Educativa, 8, 108-127.

GonZÁlEz, Y. (2015). El papel del docente en la educación para la sexualidad: algunas reflexiones en el proceso educativo escolar. Revista Actualidades Investigativas en Educación, 15(3), 1-15. 
Grau, C., Fernández, M. y Cuesta, J. L. (2015). El síndrome del cromosoma X frágil: fenotipo conductual y dificultades de aprendizaje. Siglo Cero, 46(4), 25-44.

Hannah, L. y STAGG, S. (2016). Experiences of sex education and sexual awareness in young adults with autism spectrum disorder. Journal of Autism and Developmental Disorders, 46(12), 3678-3687.

Haracopos, D. y Pedersen, L. (1992). Sexuality and autism: a nationwide survey in Denmark. Recuperado de https://www.autismuk.com/autism/sexuality-and-autism/sexualityand-autism-danish-report/.

Hatton, S. y Tector, A. (2010). Sexuality and relationship education for young people with autistic spectrum disorder: curriculum change and staff support. British Journal of Special Education, 37(2), 69-76.

Holmes, L., Himle, M. B. y Strassberg, D. S. (2016). Parental romantic expectations and parent-child sexuality communication in autism spectrum disorders. The International Journal of Research and Practice, 20(6), 687-699.

Jiménez, B. y Gutiérrez, V. (2006). Autismo y sexualidad en Tenerife. Inclusión y diversidad. Innovaciones y experiencias. Málaga: Aljibe.

Kalyva, E. (2010). Teachers' perspectives of the sexuality of children with autism spectrum disorders. Research in Autism Spectrum Disorders, 4(3), 433-437.

Koller, R. (2000). Sexuality and adolescents with autism. Sexuality and Disability, 18, 125-135.

Lassalle, A., Zürcher, N. R., Hippolyte, L., Billstedt, E., Porro, C. A., Benuzzi, F., Solomon, P., Prkachin, K. M., Lemonnier, E., Gillberg, C., Johnels, J. A. y HadjikHANI, N. (2018). Effect of visual stimuli of pain on empathy brain network in people with and without autism spectrum disorder. European Journal of Neuroscience, 48(6), 2333 2342. https://doi.org/10.1111/ejn.14138.

López, F. (1990). Educación sexual. Madrid: Fundación Universidad-Empresa.

López, N. (2015). La educación afectiva y sexual en el actual y futuro profesorado (Tesis doctoral). Universidad de Las Palmas de Gran Canaria, España.

Lozano, J., Alcaraz, S. y Bernabeu, M. (2012). Competencias emocionales del alumnado con trastornos del espectro autista en un aula abierta específica de educación secundaria. Aula Abierta, 40(1), 15-26.

Lozano, J., Castillo, I. S., García, C. у Motos, E. (2013). El desarrollo de habilidades emocionales y sociales en alumnado con trastorno del espectro autista: una investigación colaborativa en Educación Infantil y Primaria. Revista DIM: Didáctica, Innovación y Multimedia, 26, 1-11.

Ludlow, B. (1991). Contemporary issues in sexuality and mental retardation. Advances in Mental Retardation and Developmental Disabilities, 4, 1-26.

Menzabin, P. y Stokes, M. A. (2011). Self-assessed sexuality in young adults with HighFunctioning Autism. Research in Autism Spectrum Disorders, 5, 614-621.

Mira, R. y Grau, C. (2017). Los sistemas alternativos y aumentativos de comunicación (SAAC) como instrumento para disminuir conductas desafiantes en el alumnado con TEA: estudio de un caso. Revista Española de Discapacidad, 5(1), 113-132.

Moro, L., Jenaro, C. y Solano, M. (2015). Miedos, esperanzas y reivindicaciones de padres de niños con TEA. Siglo Cero, 46(4), 7-24.

Mulas, F., Ros-Cervera, G., Millá, M. G., Etchepareborda, M. C., Abab, L. y Téllez de Menese, M. (2010). Modelos de intervención en niños con autismo. Revista de Neurología, 50(3), 77-84.

Newport, J. y Newport, M. (2002). Autism-Asperger's and sexuality: puberty and beyond. Arlington, Texas: Future Horizons. 
Oliva, M. (2012). La educación sexual integral en el aula ¿derecho u obligación? Novedades Educativas, 259, 18-19.

Otтoni, A. C. V. y MaiA, A. C. B. (2019). Considerações sobre a sexualidade e educação sexual de pessoas com trastorno do espectro autista. Revista Ibero-Americana de Estudos em Educação, Araraquara, 14(2), 1265-1283. https://doi.org/10.21723/riaee.v14iesp.2.12575.

Padrón, M. ${ }^{a}$ M., Fernández, L., Infante, A. y París, A. (2009). Libro Blanco sobre Educación Sexual de la provincia de Málaga. Málaga: Área de Juventud, Deportes y Formación de la Diputación de Málaga.

Pask, L., Hughes, T. L. y Sutton, L. R. (2016). Sexual knowledge acquisition and retention for individuals with autism. International Journal of School and Educational Psychology, 4(2), 86-94.

Pellejero, L. y Torres, B. (2011). La educación de la sexualidad: el sexo y el género en los libros de texto de Educación Primaria. Revista de Educación, 354, 399-427.

Rubin, G. (1984). Reflexionando sobre el sexo: notas para una teoría radical de la sexualidad. Biblioteca Virtual de Ciencias Sociales, 1-59. Recuperado de http://www.museo-etnografico.com/pdf/puntodefuga/150121gaylerubin.pdf.

Sevlever, M., Roth, M. y Gillis, J. (2013). Sexual abuse and offending in autism spectrum disorders. Sexuality and Disability, 31(2), 189-200.

Stokes, M., Newton, N. y Kaur, A. (2007). Stalking, and social, and romantic functioning among adolescents and adults with autism spectrum disorder. Journal of Autism and Developmental Disorders, 37, 1969-1986. https://doi.org/10.1007/s10803-006-0344-2.

Travers, J. y Tincani, M. (2010). Sexuality education for individuals with autism spectrum disorders: critical issues and decision making guidelines. Education and Training in Autism and Developmental Disabilities, 45(2), 284-293.

UNESCO (2014). Educación integral de la sexualidad: conceptos, enfoques y competencias. Oficina Regional de Educación para América Latina y el Caribe. Recuperado de http:// unesdoc.unesco.org/images/0023/002328/232800S.pdf.

Venegas, M. (2017). Coeducar las relaciones afectivo-sexuales para promover la igualdad sexual y de género y la justicia social. Revista Internacional de Educación para la Justicia Social (RIEJS), 6(2), 13-28. https://doi.org/10.15366/riejs.

Walters, J. B., Hughes, T. L., Sutton, L. R., Marshall, S. N., Crothers, L. M., Lehman, C., Paserba, D., Talkington, V., Taormina, R. y Huang, A. (2013). Maltreatment and depression in adolescent sexual offenders with an autism spectrum disorder. Journal of Child Sexual Abuse, 22(1), 72-89.

World Association For Sexual Health (WAS) (2015). WAS promoting global sexual health, sexual rights and universal sexuality education. Boletin, 12(1).

Zigler, E. y Hodapp, R. (1986). Understanding mental retardation. New York: Cambridge University Press. 


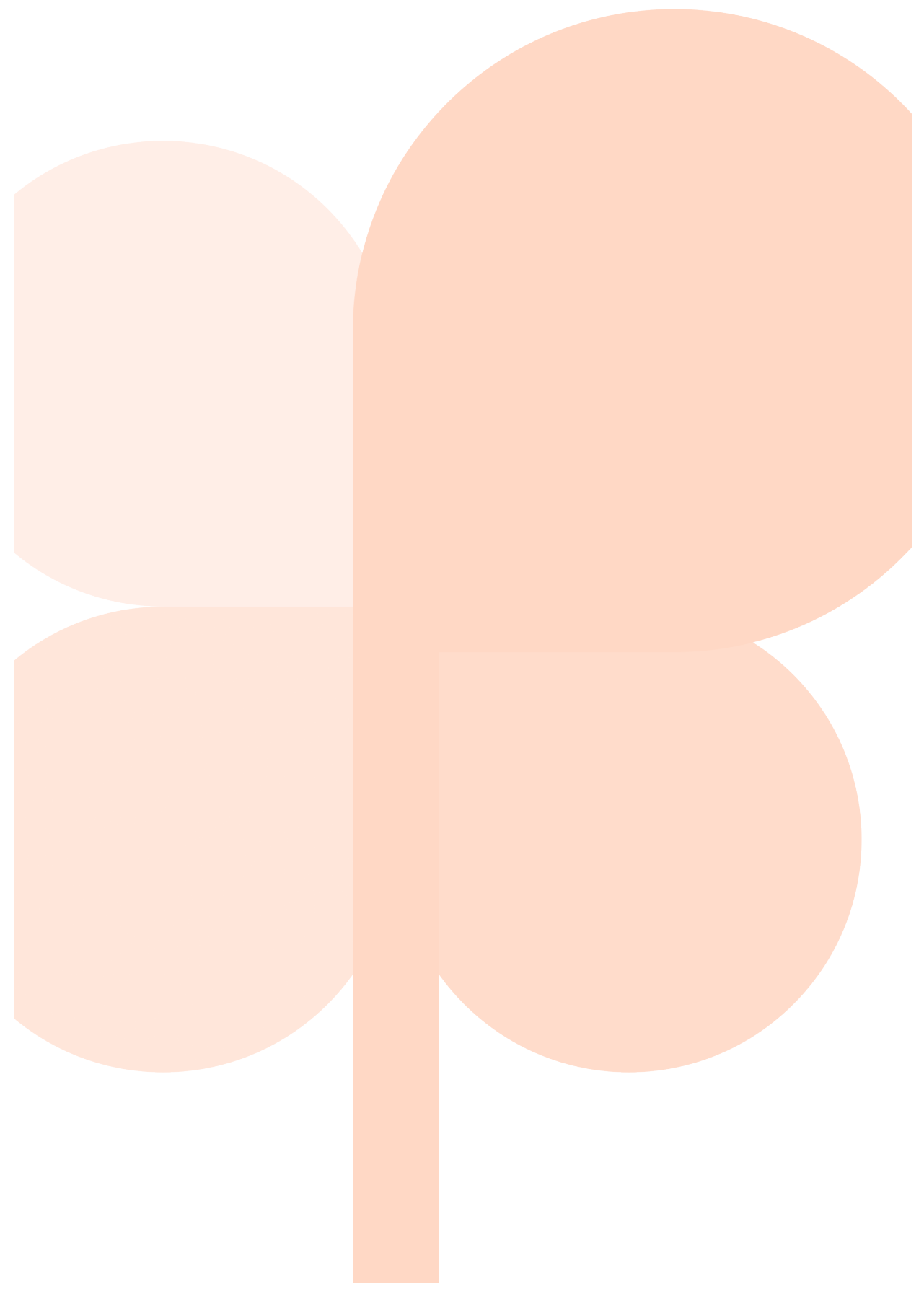

